\title{
Defect Verlinde Formula for Edge Excitations in Topological Order
}

\author{
Ce Shen ${ }^{*}$ and Ling-Yan Hung ${ }^{\dagger}$ \\ State Key Laboratory of Surface Physics, Fudan University, 200433 Shanghai, China, \\ Department of Physics and Center for Field Theory and Particle Physics, Fudan University, Shanghai 200433, China, \\ and Institute for Nanoelectronic Devices and Quantum Computing, Fudan University, 200433 Shanghai, China
}

(Received 20 February 2019; published 1 August 2019)

\begin{abstract}
We revisit the problem of boundary excitations at a topological boundary or junction defects between topological boundaries in nonchiral bosonic topological orders in $2+1$ dimensions. Based on physical considerations, we derive a formula that relates the fusion rules of the boundary excitations and the "halflinking" number between condensed anyons and confined boundary excitations. This formula is a direct analogue of the Verlinde formula. We also demonstrate how these half-linking numbers can be computed in explicit Abelian and non-Abelian examples. As a fundamental property of topological orders and their allowed boundaries, this should also find applications in the search for suitable platforms realizing quantum computing devices.
\end{abstract}

DOI: 10.1103/PhysRevLett.123.051602

Topological orders contain many intricate structures, such as fusion and braiding among anyons. This topic has been studied in depth in the past few decades. More recently, it has been realized that topological interfaces connecting different topological orders contain various interesting structures [1-5]. These interfaces can be described by the physics of anyon condensation. Excitations localized at the interface (confined anyons) satisfy interesting (and generically noncommutative) fusion rules. These objects are also intimately related to topological defect lines in conformal field theories (CFT's), which have been extensively studied e.g., in [6-12]. They form a fusion tensor category that does not have a well-defined braiding structure, but they do admit a nontrivial half-linking with condensed anyons.

Eliciting their properties gives us important new insights into the fundamental structure of topological field theories and the relationships between them.

Topological orders have also found applications in quantum computing, given their robustness against decoherence, and it has been proposed in the literature that topological defects might in fact be a more convenient candidate to realize universal computing [13]. Therefore, the fusion and half-linking properties mentioned above acquire practical significance.

In this paper, we find that the set of physical datanamely, the fusion rules of the defects and half-linkingare related in a way directly analogous to the Verlinde

Published by the American Physical Society under the terms of the Creative Commons Attribution 4.0 International license. Further distribution of this work must maintain attribution to the author(s) and the published article's title, journal citation, and DOI. Funded by SCOAP ${ }^{3}$. formula. We consider confined anyons in a given boundary condensate, which we subsequently generalize to confined anyons localized at the junction between two different boundary condensates. This method would have applications both in the study of interfaces in CFT and also in experimental realizations of defect-based topological quantum computations [14].

Gapped boundaries can be described by anyon condensation $[1,3,15-22]$. For a given nonchiral bulk phase $\mathcal{B}$ in $2+1$ dimensions, there could be multiple different gapped boundaries, each characterized by a distinct pattern of anyon condensation.

The bulk phase is describable by a modular tensor category $\mathcal{B}$. The basic physical data are the distinct simple topological sectors, or anyons $a$ with quantum dimension $d_{a}$. These sectors can fuse, i.e.,

$$
a \otimes b=\oplus_{c} N_{a b}^{c} c
$$

and these fusion rules are associative. The self-statistics of the anyons are encoded in the eigenvalues of the so-called modular $T$ matrix [23], and their mutual statistics are encoded in the modular $S$ matrix, $S_{a b}$ [23]. The Verlinde formula is a renowned relation between $N_{a b}^{c}$ and $S_{a b}$ [24],

$$
N_{a b}^{c}=\sum_{d} \frac{S_{a d} S_{b d}\left(S^{-1}\right)^{d c}}{S_{0 d}}
$$

The most important physical data characterizing a given pattern of anyon condensation corresponding to a gapped boundary are the set of condensed anyons, i.e., the condensate $\mathrm{C}$. They form a Lagrangian algebra in the bulk phase $\mathcal{B}$. The condensate would behave like the trivial 
sector in the condensed phase. In the case of a gapped boundary, the condensed phase is the trivial topological order with only one (trivial) sector. Anyons not belonging to the condensate are "confined," and they would correspond to boundary excitations as they approach the boundary. Not every confined anyon in the bulk corresponds to a distinct boundary excitation since they are identified if they are related by fusion with one of the condensed anyons $c$. To be precise, the relation between bulk anyons $a$ and boundary excitations $x$ can be expressed using the $W$ matrix $[15,16]$

$$
a=\oplus_{x} W_{a x} x,
$$

where $W_{a x}$ is a positive integer that gives the "multiplicity" of $a$ decomposing into $x$ [25]. If $a$ is part of the condensate $\mathrm{C}$, then $W_{a 0} \neq 0$. And it follows that

$$
\mathbf{C}=\oplus_{c} W_{c 0} c .
$$

In the following, when referring to an anyon that is condensed, we loosely use the notation $c \in \mathrm{C}$.

The decomposition (3) commutes with fusion, i.e.,

$$
\sum_{c} N_{a b}^{c} W_{c z}=\sum_{x, y} W_{a x} W_{b y} n_{x y}^{z}
$$

where $n_{x y}^{z}$ is the fusion coefficient of the boundary excitations corresponding to confined anyons in the condensate.

Boundary excitations are confined anyons that are stuck at the boundary; they are not capable of getting to the condensed phase without creating a trail of excitations. Condensed anyons, on the other hand, can freely pass through the boundary. This idea suggests a natural topological number characteristic of the boundary condensate. Namely, consider creating a boundary anyon $x$ and its antiparticle. Now, a condensed anyon $c$ is created from the boundary, moved around $x$ in the bulk, and finally annihilated at the boundary. Then, we also annihilate the pair of $x$ 's [see Fig. 1(a)]. This is, up to normalization, closely related to the half-linking mentioned in the context of gapped boundaries of Abelian Chern-Simons theory [26]. Here, we generalize the discussion to non-Abelian systems, and we denote this quantity by

$$
\gamma_{x c_{(i, j)}}
$$

Here, there are extra indices $i, j$ if $W_{c 0} \geq 2$. Then, one has to specify the "condensation channel" of the condensed anyon $c$, i.e., $1 \leq i \leq W_{c 0}$, where it is created and annihilated at the boundary. We note that $[3,22,27]$

$$
\sum_{c \in \mathcal{B}} W_{c 0} W_{c 0}=N_{x}
$$

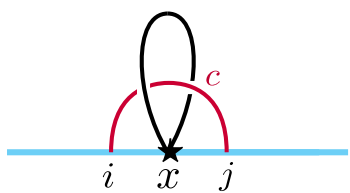

(a)

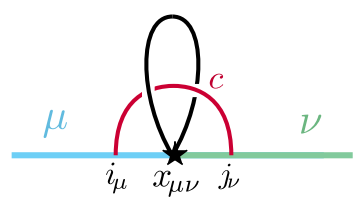

(b)
FIG. 1. (a) Half-linking $\gamma_{x c_{(i, j)}}$ with boundary excitation $x$ and condensed anyon $c$. Here, $i$ and $j$ label the condensation channel. (b) Half-linking $\gamma_{x c_{\left(i_{\mu}, j_{\nu}\right)}}^{\left.(\mu \mid)_{\nu}\right)}$ with defect $x_{\mu \nu}$ living at the junction between two gapped boundaries $\mu$ and $\nu$, while the shared condensed anyon $c \in \mathrm{C}_{\mu} \cap \mathrm{C}_{\nu}$.

where $N_{x}$ is the number of distinct simple boundary excitations. This condition is necessary for $\gamma_{x c_{(i, j)}}$ to be invertible as an $N_{x} \times N_{x}$ matrix. Moreover, given two different gapped boundaries labeled $\mu, \nu$, which are characterized by $W^{(\mu)}$ and $W^{(\nu)}$, respectively, the number $N_{\mu \nu}$ of distinct species of anyons that are localized at the junction of the two boundaries is given by

$$
N_{\mu \nu}=\sum_{c \in \mathcal{B}} W_{c 0}^{(\mu)} W_{c 0}^{(\nu)}
$$

This case is depicted in Fig. 1(b).

By considering the half-linking of a condensed anyon around two boundary excitations $x, y$ before and after the fusion, one can deduce an important relation between the half-linking number and the fusion coefficients $n_{x y}^{z}$ of boundary excitations. A graphical representation of the processes is given in Fig. 2. Note that we have made use of the fact that the worldline of a condensed anyon can be deformed and can break up at the boundary. It leads to the following equation:

$$
n_{x y}^{z}=\sum_{c} \sum_{i, j, l, k} \gamma_{x c_{(i, j)}}\left(M_{c}^{-1}\right)_{j l} \gamma_{y c_{(l, k)}} \gamma_{\left.c_{(k, i)}\right)}^{-1}
$$

Here, the half-linking is an invertible matrix, such that $\gamma^{-1}$ satisfies

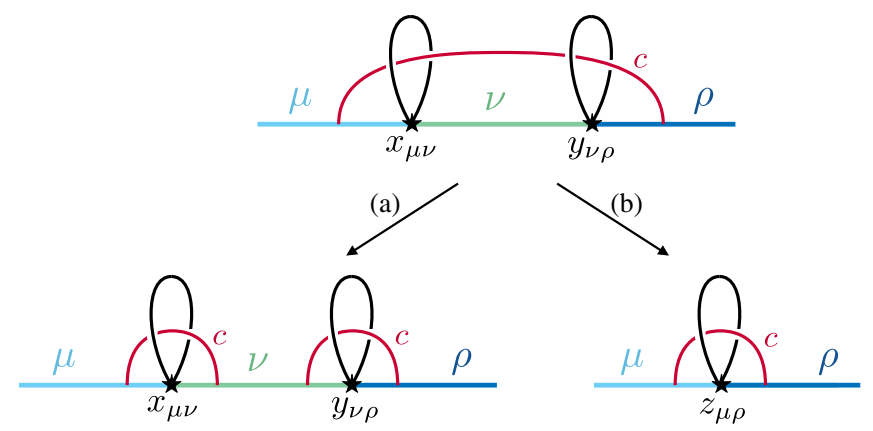

FIG. 2. (a) Deforming the condensed $c$ anyon line yields two half-links. (b) Shrinking the middle $\nu$ boundary fuses the two defects $x_{\mu \nu}$ and $y_{\nu \rho}$. 


$$
\begin{gathered}
\sum_{c} \sum_{i, j} \gamma_{\left.x c_{(i, j)}\right)} \gamma_{c_{(j, i)} y}^{-1}=\delta_{x y}, \\
\sum_{x} \gamma_{c_{\left(j^{\prime}, i^{\prime}\right)}^{-1}}^{-1} \gamma_{x c_{(i, j)}}=\delta_{c, c^{\prime}} \delta_{i, i^{\prime}} \delta_{j, j^{\prime}} .
\end{gathered}
$$

We have introduced a proportionality matrix $\left(M_{c}^{-1}\right)_{j l}$ that is closely related to the boundary $3 j$ symbols. They describe the deformation of a condensed anyon line so that it breaks up at the boundary [27]. This coefficient can be determined in terms of the half-linking matrices for the given boundary. Consider winding a condensed anyon $c$ around vacuum $x=y=0$. Then, Eq. (9) implies

$$
\left(M_{c}\right)_{i j}=\gamma_{0 c_{(i, j)}},
$$

where $\gamma_{0 c_{(i, j)}}$ is taken to be invertible as a matrix with indices $i, j$ [28].

This case is the analogue of the Verlinde formula for boundary excitations. We note that this formula is closely related to the discussion of defect operators in the context of CFT, where boundary excitations correspond to defect line operators in a given modular invariant CFT $[6,9]$. This modular invariant is in direct correspondence with the Lagrangian algebra characterizing the gapped boundary. Here, we arrive at the result via a different route that is based on the physical process in the $2+1$-dimensional system.

However, this result can be further generalized. Consider two gapped boundaries $\mu, \nu$ joining at a junction, with corresponding sets of boundary excitations $X_{\mu}$ and $X_{\nu}$, respectively. Among those confined anyons, some are confined in both phases $\mu$ and $\nu$. They are thus localized at the junction connecting the two boundaries $\mu, \nu$. We denote the set of excitations by $X_{\mu \nu}$. Among condensed anyons in $\mu, \nu$, there are also shared condensed anyons $c \in \mathrm{C}_{\mu} \cap \mathrm{C}_{\nu}$ that can be freely created and annihilated at both boundaries. We can thus define a half-linking between $c$ and $x \in X_{\mu \nu}$,

$$
\gamma_{x c_{\left(i_{\mu}, i_{i}\right)}}^{(\mu \mid \nu)}, \quad 1 \leq i_{\alpha} \leq W_{c 0}^{(\alpha)}, \quad \alpha \in\{\mu, \nu\},
$$

which reduces to (6) when $\mu=\nu$.

Now, consider three adjacent gapped boundaries $\mu, \nu, \rho$, where $\mu$ is connected to $\nu$ and $\nu$ connected to $\rho$. Since these boundaries are gapped, we can adiabatically compress the sizes of these boundaries. Therefore, excitations localized at different junctions $x_{\mu \nu}$ and $y_{\nu \rho}$ can also be fused to obtain localized excitations between $\mu$ and $\rho$, as the $\nu$ boundary is compressed to vanishing size; i.e., the fusion rules are generalized to include

$x \otimes y=\bigoplus_{z} n_{x y}^{z} z, \quad x \in X_{\mu \nu}, \quad y \in X_{\nu \rho}, \quad z \in X_{\mu \rho}$.

Then, we consider the half-link of a condensed anyon $c \in \mathrm{C}_{\mu} \cap \mathrm{C}_{\rho}$ around $x$ and $y$. One can either deform the condensed anyon line $c$ so that it becomes two half-links, separately around $x$ and $y$, or one can first fuse $x$ and $y$ before computing the half-linking number with the fusion product. This process is illustrated in Fig. 2 and gives another relation between the half-linking numbers and the fusion coefficients:

$$
\begin{aligned}
n_{x y}^{z} & =\sum_{c} \sum_{i_{\mu}, i_{\nu}, i_{\nu}^{\prime}, i_{\rho}} \gamma_{x c_{\left(i_{\mu}, i_{\nu}\right)}}^{(\mu \mid \nu)}\left(M_{c}^{\nu}\right)_{i_{\nu} i_{\nu}^{\prime}}^{-1} \gamma_{y c}^{(\nu \mid \rho)}\left(c_{\left(i_{\nu}^{\prime}, i_{\rho}\right)}\left(\gamma^{(\mu \mid \rho)}\right)_{c_{\left(i_{\rho}, i_{\mu}\right)} z}^{-1}\right. \\
& =\sum_{c} \gamma_{x c}^{(\mu \mid \nu)}\left(M_{c}^{\nu}\right)^{-1} \gamma_{y c}^{(\nu \mid \rho)}\left(\gamma^{(\mu \mid \rho)}\right)_{c z}^{-1}
\end{aligned}
$$

where we have included a subscript $\nu$ for the matrix $M_{c}$ and simplified our notation in the last line to keep the condensation channel indices $i_{\alpha}$ implicit. Equations (9) and (15) are the main results of this paper. To the best of our knowledge, Eq. (15), together with accompanying examples that we discuss, appears here for the first time in the mathematics literature.

The formula above is only useful if we can compute these matrices. We make an observation here. Consider putting a topological order $\mathcal{B}$ on a cylinder, with the top boundary labeled $\mu$ and the bottom boundary labeled $\nu$. The ground-state degeneracy is given by $N_{\mu \nu}$ in (8). There are two possible sets of basis states, namely, states corresponding to confined anyons winding the noncontractible loop, labeled by $\left|\varsigma_{x}\right\rangle$, and condensed anyons connecting the top and bottom boundaries, labeled by $\left.\mid \begin{array}{l}i_{\mu} \\ \uparrow_{c}^{c} \\ i_{\nu}\end{array}\right)$ One finds that the change of basis is effected by the half-linking numbers:

$$
\left|\bigotimes_{x}\right\rangle=\sum_{c \in \mathrm{C}_{\mu} \cap C_{\nu}} \sum_{i_{\mu}, i_{\nu}} \gamma_{x c_{\left(i_{\mu}, i_{\nu}\right)}^{(\mu \mid)}}^{\left(\uparrow_{c}\right.}\left|\begin{array}{c}
i_{\mu} \\
i_{\nu}
\end{array}\right\rangle
$$

This method gives us a very practical way of computing these matrices in explicit models, such as Abelian ChernSimons theories, and lattice models. We discover useful relations from these exercises that can be applied in other, more general situations. Moreover, given that it is a basis transformation, it is expected to be a unitary transformation. This is indeed the case in all the examples we encountered. In the following, we therefore write

$$
\left(\gamma^{(\mu \mid \nu)}\right)_{c x}^{-1}=\overline{\gamma_{x c}^{(\mu \mid \nu)}}
$$

We first consider the case of a single boundary where the corresponding anyon condensation multiplicity $W_{c 0} \leq 1$ for a given topological boundary. In this case, we can provide an alternative proof of (9), where no extra multiplicity indices $c_{i}$ are needed. Using Eqs. (2) and (5), we obtain 


$$
n_{x y}^{z}=\sum_{c} \frac{V_{x c} V_{y c} V_{c z}^{-1}}{S_{0 c}}, \quad V_{c x}^{-1}=\sum_{a} \overline{S_{a c}} W_{a x}
$$

producing an alternate form of the defect Verlinde formula for a given boundary. Suppose

$$
\frac{\gamma_{x c}}{\gamma_{0 c}}=V_{c x}^{-1}, \quad \gamma_{0 c}=\sqrt{S_{0 c}} .
$$

Substituting into (18), we recover (9). The relation (19) is an empirical observation based on Abelian Chern-Simons theories, the quantum double model $D\left(S_{3}\right)$, and also generic tensor product theories $\mathcal{B}=\mathcal{D} \otimes \overline{\mathcal{D}}$, where $\mathcal{D}$ is some modular tensor category and $\overline{\mathcal{D}}$ its time reversal. In this last case, "diagonal condensation" always gives an allowed gapped boundary, where every anyon of the form $(a \bar{a})$ condenses, for $a \in \mathcal{D}$. The half-linking matrix that defines the transformation (16) would coincide with the modular matrix $S^{\mathcal{D}}$ of $\mathcal{D}$. This result follows from the fact that the cylinder with diagonal condensates on both edges can be unfolded into the phase $\mathcal{D}$ covering a torus. Given these infinite classes of theories, we conjecture that (19) is true, in general, where $W_{c 0} \leq 1$.

Explicit examples are relegated to the Supplemental Material [29].

More interestingly, in non-Abelian systems, one could have boundaries characterized by anyon condensation with $W_{c 0}>1$. We illustrate this using the quantum double model $D(G)[30,31]$, which is a gauge theory with gauge group $G$. Different anyonic sectors correspond to different conjugacy classes and irreducible representations of their centralizers. We particularly consider non-Abelian groups. Among the gapped boundaries, one corresponds to the socalled electric condensate, where anyons corresponding to all the representations of $G$ condense. A representation $R$ of dimension $d_{R}$ would carry a condensation multiplicity $W_{R 0}=d_{R}$ [32]. There are $|G|$ types of defects (confined anyons) in the condensate, corresponding to the magnetic charges of the gauge group $G$, which are labeled simply by the group elements $g \in G$. While magnetic anyons in the bulk phase are labeled by conjugacy classes of the gauge group, they split up into individual group elements in an electric condensate. On a cylinder with electric boundaries, the two sets of basis states discussed in (16) are

$$
\begin{gathered}
\left|\bigodot_{x}\right\rangle \rightarrow|g\rangle, \quad g \in G \\
\left|\begin{array}{c}
j \\
\uparrow_{c} \\
i
\end{array}\right\rangle \rightarrow\left|R, R_{i}, R_{j}\right\rangle, \quad 1 \leq R_{i, j} \leq d_{R} .
\end{gathered}
$$

In this case, one can readily work out the transformation matrix between these basis states, which, as expected, is the direct analogue of the transformation between group and representation basis states in a lattice gauge theory [33]:

$$
|g\rangle=\sum_{R, R_{i}, R_{j}} \sqrt{\frac{d_{R}}{|G|}} \rho_{R_{j}, R_{i}}^{R}\left(g^{-1}\right)\left|R, R_{i}, R_{j}\right\rangle .
$$

Now, substituting into (9), we have

$$
\begin{aligned}
N_{g_{1} g_{2}}^{g_{3}} & =\sum_{R} \frac{d_{R}}{|G|} \chi^{R}\left(g_{1}^{-1} g_{2}^{-1} g_{3}\right) \\
& =\delta_{g_{2} \times g_{1}, g_{3}} .
\end{aligned}
$$

We have used (12) above, which reduces here to

$$
M_{R}=\sqrt{\frac{d_{R}}{|G|}} \mathbb{I}_{R} .
$$

The fusion of the defects coincide with the group product rule, which gives a natural realization of a noncommutative fusion ring envisaged in the CFT literature [7]. Invertible Verlinde lines in Rational Conformal Field Theory (RCFT) are also expected to satisfy a fusion rule that is equivalent to group multiplication [11]. We derive these rules using the defect Verlinde formula here.

We would now like to discuss explicit examples realizing (15).

To be explicit, there is a class of Abelian theories whose boundaries can be easily described. These Abelian theories are equivalent to the quantum doubles $D(G)$, where $G$ is a finite Abelian group. The quantum dimension of the theory is given by $D=|G|$. Each boundary is characterized by an Abelian subgroup $K \subset G$, which is in direct correspondence with a set of condensed anyons. With details given in the Supplemental Material [29], we find that the halflinking matrix at a junction between two boundaries characterized by two subgroups $K_{\mu, \nu}$ is given by

$$
\gamma_{x c}^{(\mu \mid \nu)}=\frac{1}{\sqrt{D}} \frac{\sqrt{\left|K_{\mu}\right| \cdot\left|K_{\nu}\right|}}{\left|K_{\mu} \cap K_{\nu}\right|} \tilde{S}_{x^{B} c}
$$

The matrix $\tilde{S}$ is proportional to the bulk $S$-matrix,

$$
\tilde{S}_{x c}=\frac{1}{D} S_{x c}=\exp \left(2 \pi i \mathbf{l}_{c}^{T} K^{-1}\left(\mathbf{l}_{x}\right)\right)
$$

and $\mathbf{l}_{c}, \mathbf{l}_{x}$ are (two-component) integer charge vectors corresponding to the topological sectors $c$ and $x$, respectively. In (26), $x^{\mathcal{B}}$ is a representative bulk anyon chosen to satisfy $W_{x^{B} x}^{\mu}=W_{x^{\mathcal{B}} x}^{\nu}=1, x \in X_{\mu \nu}$. Substituting this into (15), it recovers the fusion rule 


$$
\begin{aligned}
\delta_{\mathbf{l}_{x}+\mathbf{l}_{y}, \mathbf{l}_{z}} & \equiv n_{x y}^{z}=\sum_{c \in \mathrm{C}_{\mu} \cap \mathrm{C}_{\nu} \cap \mathrm{C}_{\rho}} \gamma_{x c}^{(\mu \mid \nu)} M_{c}^{(\nu)-1} \gamma_{y c}^{(\nu \mid \rho) \overline{(\mu \mid \rho)}}, \\
x & \in X_{\mu \nu}, \quad y \in X_{\nu \rho}, \quad z \in X_{\mu \rho},
\end{aligned}
$$

where $C_{\mu}$ is the set of condensed anyons of the boundary $\mu$.

Let us give a non-Abelian example based on the quantum double $D\left(S_{3}\right)$. We illustrate the fusion of defects at junctions between two gapped boundaries characterized by the condensates $\mathcal{A}_{3}=A \oplus C \oplus D$ and $\mathcal{A}_{4}=$ $A \oplus F \oplus D$. (Another interesting junction between $\mathcal{A}_{1}=$ $A \oplus B \oplus 2 C$ and $\mathcal{A}_{3}=A \oplus C \oplus D$ is relegated to the Supplemental Material [29]). The half-linking matrices between various $S_{3}$ boundaries are listed in the Supplemental Material [29].

For $\mathcal{A}_{3}\left|\mathcal{A}_{4}\right| \mathcal{A}_{3}$ defect fusion, we calculate the following fusion coefficients ([see Supplemental Material [29] for $D\left(S_{3}\right)$ half-linking matrices):

$$
n_{x y}^{z}=\sum_{c \in\{A, D\}} \frac{\gamma_{x c}^{(3 \mid 4)} \gamma_{y c}^{(4 \mid 3)} \overline{\gamma_{z c}^{(3 \mid 3)}}}{\gamma_{0 c}^{(4 \mid 4)}} .
$$

Here, the $M_{c}$ matrix is simply a number $\gamma_{0 c}^{(4 / 4)}$. We can derive the fusion rules:

$$
\begin{aligned}
& \{A\}^{(3 \mid 4)} \otimes\{A\}^{(4 \mid 3)}=\{A\}^{(3 \mid 3)} \oplus\{F\}^{(3 \mid 3),} \\
& \{A\}^{(3 \mid 4)} \otimes\{B\}^{(4 \mid 3)}=\{B\}^{(3 \mid 3)} \oplus\{F\}^{(3 \mid 3)}, \\
& \{B\}^{(3 \mid 4)} \otimes\{B\}^{(4 \mid 3)}=\{A\}^{(3 \mid 3)} \oplus\{F\}^{(3 \mid 3)} .
\end{aligned}
$$

The fusion involving the defect $\{A\}$ was obtained in Ref. [34], based on a heuristic method. The complete fusion rules are now derived using the defect Verlinde formula.

ConclusionIn this paper, we have studied the boundary excitations and junction excitations at and between topological boundaries. We found that the fusion algebra of these boundary excitations can be naturally connected to the half-linking matrix, in direct analogy to the Verlinde formula. We derived an explicit expression of this defect Verlinde formula and presented examples, including both Abelian and non-Abelian ones. This study should find numerous applications in recovering fusion algebra of defects in topological orders, which are hopeful candidates for the construction of viable quantum computing devices $[13,30,35]$.

We thank Laurent Freidel, Davide Gaiotto, Matthias Gaberdiel, Liang Kong, Yidun Wan, Xiao-Gang Wen, and Gabriel Wong for discussions. Part of this work was completed during the authors' visit to the Perimeter Institute as part of the Emmy-Noether Fellowship program. L. Y. H. acknowledges the support of Fudan University and the Thousands Young Talents Program. This work is supported by the NSFC Grant No. 11875111. *scbebetterme@gmail.com

†lyhung@ fudan.edu.cn

[1] L. Kong, Nucl. Phys. B886, 436 (2014).

[2] J. Fuchs, C. Schweigert, and A. Valentino, Commun. Math. Phys. 321, 543 (2013).

[3] T. Lan, J. C. Wang, and X.-G. Wen, Phys. Rev. Lett. 114, 076402 (2015).

[4] J. Wang, X.-G. Wen, and S.-T. Yau, arXiv:1602.05951.

[5] J. Wang, X.-G. Wen, and S.-T. Yau, arXiv:1901.11537.

[6] V. B. Petkova and J.-B. Zuber, Phys. Lett. B 504, 157 (2001).

[7] V. Petkova and J.-B. Zuber, in Progress in Mathematical Physics, edited by M. Kashiwara and T. Miwa (Birkhäuser, Boston, 2002), Vol. 23, p. 415, https://link.springer.com/ chapter/10.1007\%2F978-1-4612-0087-1_15.

[8] M. R. Gaberdiel and T. Gannon, Nucl. Phys. B639, 471 (2002).

[9] J. Fuchs, I. Runkel, and C. Schweigert, Applied Categorical Structures 16, 123 (2008).

[10] D. Gaiotto, arXiv:1404.0332.

[11] C.-M. Chang, Y.-H. Lin, S.-H. Shao, Y. Wang, and X. Yin, J. High Energy Phys. 01 (2019) 026.

[12] G. Pradisi, A. Sagnotti, and Y. S. Stanev, Phys. Lett. B 381, 97 (1996).

[13] I. Cong and Z. Wang, arXiv:1710.07197.

[14] Z. Luo, J. Li, Z. Li, L.-Y. Hung, Y. Wan, X. Peng, and J. Du, Nat. Phys. 14, 160 (2018).

[15] F. A. Bais, B. J. Schroers, and J. K. Slingerland, Phys. Rev. Lett. 89, 181601 (2002).

[16] F. A. Bais and J. K. Slingerland, Phys. Rev. B 79, 045316 (2009).

[17] A. Kitaev and L. Kong, Commun. Math. Phys. 313, 351 (2012).

[18] M. Barkeshli, C.-M. Jian, and X.-L. Qi, Phys. Rev. B 88, 241103(R) (2013).

[19] M. Barkeshli, C.-M. Jian, and X.-L. Qi, Phys. Rev. B 88, 235103 (2013).

[20] M. Levin, Phys. Rev. X 3, 021009 (2013).

[21] L.-Y. Hung and Y. Wan, J. High Energy Phys. 07 (2015) 120.

[22] L.-Y. Hung and Y. Wan, Phys. Rev. Lett. 114, 076401 (2015).

[23] J. B. B. a. A. Kirillov, Lectures on Tensor Categories and Modular Functors, University Lecture Series (American Mathematical Society, Providence, 2001).

[24] E. Verlinde, Nucl. Phys. B300, 360 (1988).

[25] This should be contrasted with the $W$ matrix discussed e.g., in Ref. [3], in which the $x$ indices contain only "unconfined" sectors.

[26] A. Kapustin and N. Saulina, Nucl. Phys. B845, 393 (2011).

[27] I. Cong, M. Cheng, and Z. Wang, arXiv:1609.02037.

[28] From the examples we have worked with, it appears that $M_{c}$ is proportional to $\delta_{i j}$, which is explicit in the examples built from gauge theories. We also expect this to be a natural result as we break an anyon line into two at the boundary.

[29] See Supplemental Material at http://link.aps.org/ supplemental/10.1103/PhysRevLett.123.051602 for halflinking matrices of Abelian Chern-Simons theory and $D\left(S_{3}\right)$.

[30] A. Y. Kitaev, Ann. Phys. (Amsterdam) 303, 2 (2003). 
[31] S. Beigi, P. W. Shor, and D. Whalen, Commun. Math. Phys. 306, 663 (2011).

[32] T.Lan, L. Kong, and X.-G. Wen, Phys. Rev. X 8, 021074 (2018).

[33] O. Buerschaper and M. Aguado, Phys. Rev. B 80, 155136 (2009).
[34] I. Cong, M. Cheng, and Z. Wang, Phys. Rev. B 96, 195129 (2017).

[35] M. Barkeshli, C.-M. Jian, and X.-L. Qi, Phys. Rev. B 87, 045130 (2013). 